\title{
MAXIMIZING THE LIFETIME OF CLUSTERS WITH SLEPIAN-WOLF CODING
}

\author{
Tianqi Wang, Wendi Heinzelman, Alireza Seyedi, and Azadeh Vosoughi
}

Electrical and Computer Engineering Department, University of Rochester

\begin{abstract}
In this paper, we propose an iteration-free algorithm to find the optimal configuration, including transmit power and source coding rates, to maximize the lifetime of a cluster utilizing Slepian-Wolf source coding of data sent to a fusion center. Exact closed form solutions are derived when the fusion center is not energy constrained. When the fusion center is energy constrained, a near optimum solution is provided. Numerical results demonstrating the performance of the proposed algorithms are also provided.
\end{abstract}

Index Terms-Distributed source coding, Wireless sensor networks, Energy efficiency, Lifetime

\section{INTRODUCTION}

In general, there are two ways to reduce energy consumption in wireless sensor networks (WSNs): efficient communication and data compression. Among the data compression schemes, distributed source coding (DSC) in particular has great potential in WSNs [1]-[3]. DSC exploits the spatial correlation commonly found in sensed signals. With only the knowledge of the spatial correlation between their signals, the sensor nodes can compress their data using DSC without communicating each other.

Slepian-Wolf coding, which is concerned with lossless DSC of two correlated discrete sources, has recently been utilized to address the energy efficient gathering of correlated data in WSNs using mathematical optimization techniques [2][4][5]. Researchers have abstracted the wireless media as a graph with fixed cost per information bit, and then jointly optimized the data gathering tree and the rate allocation across the source nodes. In practice, however, different communication constraints (e.g., network topology, energy distribution) can significantly alter the communication cost per information bit. Consequently, when considering minimization of consumed energy, it is essential that the communication techniques are considered in conjunction with the compression techniques.

In this paper, we propose an iteration-free solution to maximize the amount of data gathered during the cluster lifetime with Slepian-Wolf coding. The optimization is conducted on both the source coding and the communication sides. The closed-form solutions for joint optimal transmit powers and Slepian-Wolf coding rates are provided.

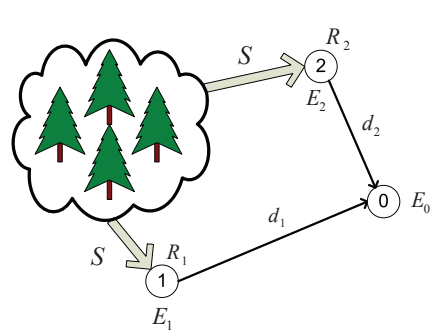

Fig. 1. The 2-node Slepian-Wolf coding scheme

\section{PROBLEM DEFINITION}

Fig. 1 shows a typical Slepian-Wolf coding scheme with 2 sensor nodes that send data to a fusion center. During the lifetime of this cluster, each sensor senses $S$ samples. The lifetime of the cluster is defined as the time duration elapsed from the beginning to the time when the fusion center or both sensor nodes die.

We denote the source coding rate at node $i$ with $R_{i}$. The Slepian-Wolf theorem states that if $R_{1} \geq H\left(X_{1} \mid X_{2}\right), R_{2} \geq$ $H\left(X_{2} \mid X_{1}\right), R_{1}+R_{2} \geq H\left(X_{1}, X_{2}\right), X_{1}$ and $X_{2}$ are reconstructible perfectly at the fusion center. Here $H(\cdot)$ represents the entropy function, and $X_{i}$ is the source random variable (sample) sensed at node $i$ [6].

Assume that sensor node $i$ can transmit for time duration $T_{i}$, and it transmits $S R_{i}$ data bits to the fusion center. Since both sensors observe the same number of samples, we have

$$
B T_{i} \log \left(1+\frac{P_{i}}{d_{i}^{n} P_{N}}\right) \geq S R_{i},
$$

where $B$ is the bandwidth, $P_{i}$ is the transmit power of node $i, d_{i}$ is the transmission distance from node $i$ to the fusion center, $n$ is the path loss exponent and $P_{N}$ is the additive white gaussian noise (AWGN) power. The inequality of the above equation comes from Shannon's channel capacity theorem [6]. Therefore, the transmit power of node $i$ is bounded by

$$
P_{i} \geq\left(2^{\frac{S R_{i}}{B T_{i}}}-1\right) d_{i}^{n} P_{N} .
$$

That is, the lower bound on the transmit power of node $i$ is determined by its time duration $T_{i}$ and $S R_{i}$.

The energy constraints at the sensors are $T_{i}\left(P_{i}+P_{C T}\right) \leq$ $E_{i}$, where $E_{i}$ denotes the residual energy at node $i$ and $P_{C T}$ is the circuit power consumption at the sensor nodes. To facilitate our analysis, we utilize (2) to relax the energy 
constraints. That is, we assume the lower bounds on transmit powers are achievable. Therefore, the sensor nodes' energy constraints become $T_{i}\left[\left(2^{\frac{S R_{i}}{B T_{i}}}-1\right) d_{i}^{n} P_{N}+P_{C T}\right] \leq E_{i}$. The energy constraint at the fusion center is $P_{C R}\left(T_{1}+T_{2}\right) \leq E_{0}$, where $E_{0}$ is the energy available at the fusion center. $P_{C R}$ is the circuit power consumption at the fusion center and represents the power consumption for receiving data.

Under the energy and Slepian-Wolf coding constraints, the maximization of the total gathered samples during the lifetime of a cluster can be modeled as

$$
\begin{aligned}
\min & -S \\
\text { s.t. } & C_{0}: T_{i}>0, S>0 \\
& C_{1}: P_{C R}\left(T_{1}+T_{2}\right) \leq E_{0} \\
& C_{2}: T_{i}\left[\left(2^{\frac{S R_{i}}{B T_{i}}}-1\right) d_{i}^{n} P_{N}+P_{C T}\right] \leq E_{i}, \\
& C_{3}: R_{1} \geq H\left(X_{1} \mid X_{2}\right) \\
& C_{4}: \\
& C_{5}: H\left(X_{2} \mid X_{1}\right) \\
& R_{1}+R_{2} \geq H\left(X_{1}, X_{2}\right)
\end{aligned}
$$

where $C_{1}$ and $C_{2}$ are energy constraints at the fusion center and sensor nodes, respectively, and $C_{3}, C_{4}$ and $C_{5}$ are Slepian-Wolf rate constraints. Our goal is, for a given residual energy and communication environment, to find the optimum transmission time $T_{i}$, coding rates $R_{i}$, and transmit powers $P_{i}$ that maximize $S$.

\section{MAXIMIZING LIFETIME WITHOUT ENERGY CONSTRAINT AT THE FUSION CENTER}

The number of samples $S$ is a monotonically decreasing function of $R_{1}$ and $R_{2}$. Therefore, $R_{1}^{*}$ and $R_{2}^{*}$ should lie on the boundary $R_{1}+R_{2}=H\left(X_{1}, X_{2}\right)$. Thus, we always have $R_{1}^{*}+R_{2}^{*}=H\left(X_{1}, X_{2}\right)$. This observation implies that constraint $C_{5}$ is always active. When there is no energy constraint at the fusion center, the sensor nodes are then free to choose the most energy-efficient transmission method by only considering constraints $C_{2}-C_{5}$. Let us define

$$
D_{i}^{*}=\max \left\{T_{i} \log _{2}\left(1+\frac{E_{i}-P_{C T} T_{i}}{T_{i} d_{i}^{n} P_{N}}\right)\right\} .
$$

Eq. (4) represents the unconstrained maximum information bits that node $i$ can send, given that both sensors use up their energy.

Proposition 1. Without loss of generality, let us assume $D_{1} *$ is acheivable, then

$$
\begin{aligned}
R_{1}^{*} & =\max \left\{H\left(X_{1} \mid X_{2}\right), \frac{H\left(X_{1}, X_{2}\right)}{1+D_{2}^{*} / D_{1}^{*}}\right\}, \\
R_{2}^{*} & =H\left(X_{1}, X_{2}\right)-\max \left\{H\left(X_{1} \mid X_{2}\right), \frac{H\left(X_{1}, X_{2}\right)}{1+D_{2}^{*} / D_{1}^{*}}\right\}, \\
T_{1}^{*} & =\frac{E_{1}}{\frac{\ln 2\left(P_{C T}-d_{1}^{n} P_{N}\right)}{W\left(\frac{\ln 2}{2 d_{1}^{n} P_{N}}\left(P_{C T}-d_{1}^{n} P_{N}\right)\right.}+P_{C T}-d_{1}^{n} P_{N}}, \\
T_{2}^{*} & =\arg \left\{T_{2} \log _{2}\left(1+\frac{E_{2}-P_{C T} T_{2}}{T_{2} d_{2}^{n} P_{N}}\right)=D_{1}^{*} \frac{R_{2}^{*}}{R_{1}^{*}}\right\},
\end{aligned}
$$

Proof: Assume that node 1 sends $\hat{D}_{1}=S R_{1}^{*}$ bits, and node 2 sends $\hat{D}_{2}=S R_{2}^{*}$ bits. Then we have

$$
\begin{aligned}
\hat{D}_{1}+\hat{D}_{2} & =S\left(R_{1}^{*}+R_{2}^{*}\right) \\
\stackrel{a}{\Longrightarrow} S & =\left(\hat{D}_{1}+\hat{D}_{2}\right) / H\left(X_{1}, X_{2}\right), \\
\stackrel{b}{\Longrightarrow} S & =\frac{\hat{D}_{1}\left(1+\frac{R_{2}^{*}}{R_{1}^{*}}\right)}{H\left(X_{1}, X_{2}\right)}=\frac{\hat{D}_{2}\left(1+\frac{R_{1}^{*}}{R_{2}^{*}}\right)}{H\left(X_{1}, X_{2}\right)},
\end{aligned}
$$

Step $(a)$ is from the fact that $R_{1}^{*}+R_{2}^{*}=H\left(X_{1}, X_{2}\right)$. Step (b) is from the fact that $\hat{D}_{1} / \hat{D}_{2}=R_{1}^{*} / R_{2}^{*}$. Step $(a)$ implies, in the case where there is no fusion center energy constraint, we need to maximize $\hat{D}_{1}+\hat{D}_{2}$, in order to maximize the number of gathered samples. Since there is no constraint from the fusion center side, at least one of $\hat{D}_{1}$ and $\hat{D}_{2}$ should be the unconstrained maximum value from (4). Without loss of generality, we assume $\hat{D}_{1}=D_{1}^{*}$ and $\hat{D}_{2} \leq D_{2}^{*}$.

When $\hat{D}_{1}=D_{1}^{*}$ and $\hat{D}_{2} \leq D_{2}^{*}$, from step $(b)$, we have $S=D_{1}^{*}\left(1+\frac{R_{2}^{*}}{R_{1}^{*}}\right) / H\left(X_{1}, X_{2}\right)$. To maximize $S, R_{2}^{*} / R_{1}^{*}$ needs to be the largest possible value. That is $R_{1}^{*}$ has to be the smallest feasible value and $R_{2}^{*}$ is the largest feasible value. We have

$$
\begin{aligned}
D_{1}^{*} \frac{R_{2}^{*}}{R_{1}^{*}} \leq D_{2}^{*} & \Longrightarrow \frac{H\left(X_{1}, X_{2}\right)-R_{1}^{*}}{R_{1}^{*}} \leq \frac{D_{2}^{*}}{D_{1}^{*}} \\
& \Longrightarrow R_{1}^{*} \geq \frac{H\left(X_{1}, X_{2}\right)}{1+D_{2}^{*} / D_{1}^{*}}
\end{aligned}
$$

Also considering the constraint $R_{1}^{*} \geq H\left(X_{1} \mid X_{2}\right)$, we have $R_{1}^{*}=\max \left\{H\left(X_{1} \mid X_{2}\right), \frac{H\left(X_{1}, \bar{X}_{2}\right)}{1+D_{2}^{*} / D_{1}^{*}}\right\}$, and $R_{2}^{*}=$ $H\left(X_{1}, X_{2}\right)-R_{1}^{*}$.

When node 1 sends $D_{1}^{*}$, the optimal $T_{1}^{*}$ can be found from (4). The corresponding transmit time duration $T_{2}^{*}$, however, is not unique. One possible analytical solution of $T_{2}^{*}$ is

$$
T_{2}^{*}=\arg \left\{T_{2} \log _{2}\left(1+\frac{E_{2}-P_{C T} T_{2}}{T_{2} d_{2}^{n} P_{N}}\right)-D_{1}^{*} \frac{R_{2}^{*}}{R_{1}^{*}}=0\right\} . \square
$$

Proposition 1 gives the exact optimal solution of $T_{1}, T_{2}, R_{1}$, and $R_{2}$ when there is no energy constraint at the fusion center. The transmit powers can be obtained by assuming both sensor nodes use up their energy. Since the optimal solution in this case is not unique, this assumption does not affect the optimality of the solution provided by proposition 1 .

\section{MAXIMIZING LIFETIME WITH ENERGY CONSTRAINT AT THE FUSION CENTER}

When the fusion center is deployed in the field with limited energy supply, based on the amount of available energy at the fusion center, there are two possibilities.

If the residual energy at the fusion center is larger than the critical value $\hat{E}_{0}=P_{C R}\left(T_{1}^{*}+T_{2}^{*}\right)$, where $T_{1}^{*}$ and $T_{2}^{*}$ are given by (5), then this scenario is equivalent to the case when there is no energy constraint at the fusion center, as considered in Section III.

On the other hand, if $E_{0}<\hat{E}_{0}$, the total transmission time duration of the sensor nodes is limited by the fusion center energy constraint. Due to the limited transmission time budget, the sensor nodes have to send their data in

where $W(\cdot)$ is the Lambert function. 
a non-optimal way. As the available energy at the fusion center increases, thereby the total transmission time budget increases, the sensor nodes will be able to choose a more efficient transmission method. Correspondingly, the gathered data at the fusion center will increase. In this section, we focus on the optimization of a cluster where the fusion center energy constraint is the limiting factor of the cluster, or a fusion-center-limited cluster.

Given a limited transmission time budget, to send as much data as possible, the sensor nodes will use as much transmit power as possible. Therefore, both sensor nodes and the fusion center will use up their energy. This observation leads us to the following propositions:

Proposition 2. In fusion-center-limited clusters, we have 1) If $\frac{E_{1}}{d_{1}^{n}}>\frac{E_{2}}{d_{2}^{n}}$, then $R_{1}^{*}=H\left(X_{1}\right)$, and $R_{2}^{*}=$ $H\left(X_{2} \mid X_{1}\right)$.

2) If $\frac{E_{1}}{d_{1}^{n}}<\frac{E_{2}}{d_{2}^{n}}$, then $R_{1}^{*}=H\left(X_{1} \mid X_{2}\right)$, and $R_{2}^{*}=H\left(X_{2}\right)$.

3) If $\frac{E_{1}}{d_{1}^{n}}=\frac{E_{2}}{d_{2}^{n}}, R_{1}^{*}$ and $R_{2}^{*}$ can be any points on the line segment $R_{1}+R_{2}=H\left(X_{1}, X_{2}\right), R_{1}>$ $H\left(X_{1} \mid X_{2}\right), R_{2}>H\left(X_{2} \mid X_{1}\right)$.

Proof: Since the fusion center is the limiting factor, the fusion center and both sensor nodes should use up their energy. Therefore, both the fusion center and the sensor nodes' energy constraints are active:

$$
\begin{array}{r}
P_{C R}\left(T_{1}+T_{2}\right)=E_{0}, \\
T_{i}\left[\left(2^{\frac{S R_{i}}{B T_{i}}}-1\right) d_{i}^{n} P_{N}+P_{C T}\right]=E_{i} .
\end{array}
$$

From (9), we have

$$
\begin{aligned}
& \log _{2}\left(\frac{\frac{E_{i}}{T_{i}}-P_{C T}}{d_{i}^{n} P_{N}}+1\right)=\frac{S R_{i}}{B T_{i}}, \\
& \stackrel{a}{\Longrightarrow} T_{i} \log _{2}\left(\frac{E_{i}}{T_{i} d_{i}^{n} P_{N}}\right) \approx \frac{S R_{i}}{B}, \\
& \Longrightarrow T_{i} \log _{2}\left(\frac{E_{i}}{d_{i}^{n} P_{N}}\right)-T_{i} \log _{2}\left(T_{i}\right) \approx \frac{S R_{i}}{B}, \\
& \stackrel{b}{\Longrightarrow} T_{i} \approx \frac{S R_{i} / B}{\log _{2}\left(\frac{E_{i}}{d_{i}^{n} P_{N}}\right)+\frac{6}{\ln 2}},
\end{aligned}
$$

where step $(a)$ assumes that the signal to noise ratio (SNR) is much greater than 1 , and $E_{i} \gg P_{C T}$. Step $(b)$ employs the approximation $T_{i} \log _{2}\left(T_{i}\right) \approx-T_{i} \frac{6}{\ln 2}$, which becomes accurate when $T_{i} \rightarrow 0$. Define $U_{i}=\log _{2}\left(\frac{E_{i}}{d_{i}^{n} P_{N}}\right)+\frac{6}{\ln 2}$, then we have $T_{i}=\frac{S R_{i}}{B U_{i}}$. Correspondingly, we have

$$
\begin{aligned}
T_{1}+T_{2} & =\frac{S}{B}\left(\frac{R_{1}}{U_{1}}+\frac{R_{2}}{U_{2}}\right), \\
\frac{T_{1}}{T_{2}} & =\frac{R_{1} U_{2}}{R_{2} U_{1}} .
\end{aligned}
$$

The fusion center energy constraint implies

$$
P_{C R}\left(T_{1}+T_{2}\right)=E_{0} .
$$

Solving equations (11) and (13) for $S$, we have

$$
S=\frac{B E_{0}}{P_{C R}}\left(\frac{R_{1}}{U_{1}}+\frac{R_{2}}{U_{2}}\right)^{-1} .
$$

Maximizing (14) under the constraint $R_{1}+R_{2}=$ $H\left(X_{1}, X_{2}\right)$, we directly have the following results: when $U_{1}>U_{2}$, then $R_{1}^{*}=H\left(X_{1}\right)$, and $R_{2}^{*}=H\left(X_{2} \mid X_{1}\right)$; when $U_{1}<U_{2}$, then $R_{1}^{*}=H\left(X_{1} \mid X_{2}\right)$, and $R_{2}^{*}=H\left(X_{2}\right)$; when $U_{1}=U_{2}, R_{1}^{*}$ and $R_{2}^{*}$ can be any points on the line segment $R_{1}+R_{2}=H\left(X_{1}, X_{2}\right), R_{1} \geq H\left(X_{1} \mid X_{2}\right), R_{2} \geq$ $H\left(X_{2} \mid X_{1}\right)$.

Proposition 3. In fusion-center-limited clusters, the closed-form near-optimal transmit time durations are:

$$
\begin{aligned}
& T_{1}^{*}=\frac{E_{0}}{P_{C R}} \frac{R_{1}^{*} U_{2}}{R_{1}^{*} U_{2}+R_{2}^{*} U_{1}}, \\
& T_{2}^{*}=\frac{E_{0}}{P_{C R}} \frac{R_{2}^{*} U_{1}}{R_{2}^{*} U_{1}+R_{1}^{*} U_{2}},
\end{aligned}
$$

where $R_{1}^{*}$ and $R_{2}^{*}$ are determined from proposition 2 .

Proof: By solving equations (12) and (13) for $T_{1}$ and $T_{2}$, we directly have proposition 3 .

\section{NUMERICAL RESULTS}

We assume that two sensor nodes are uniformly placed within a disk, with a radius of $100 \mathrm{~m}$, centered at the fusion center. The path loss exponent is $n=4$. Also, we set $H\left(X_{1}\right)=H\left(X_{2}\right)=1$ and $H\left(X_{1} \mid X_{2}\right)=H\left(X_{2} \mid X_{1}\right)=h$. We compare the following cases: (1) $R_{1}=H\left(X_{1}\right), R_{2}=$ $H\left(X_{2}\right)$ (No Slepian-Wolf coding), optimized $T_{i}$; (2) $R_{1}=$ $H\left(X_{1} \mid X_{2}\right), R_{2}=H\left(X_{2}\right)$, optimized $T_{i}$; (3) $R_{1}=R_{2}=$ $H\left(X_{1}, X_{2}\right) / 2$, optimized $T_{i}$; (4) Jointly optimized $T_{i}$ and $R_{i}$ (analytical results P.1, P.2 and P.3.); (5) Jointly optimized $T_{i}$ and $R_{i}$ (numerical).

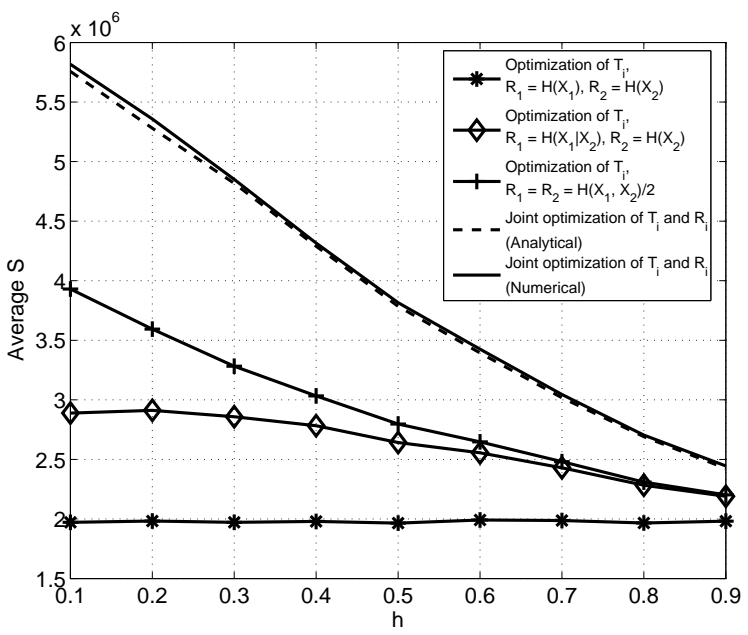

Fig. 2. Comparison of the performance in clusters with sufficient energy at the fusion center as a function of conditional entropy.

First let us consider the case where the fusion center is not the limiting factor. We fix the sensor nodes' residual energy $E_{1}=E_{2}=0.1 \mathrm{~J}$, and the fusion center residual energy $E_{0}=100 \mathrm{~J}$. In Fig. 2, we compare the average maximum number of samples with various conditional entropies in the above cases. Case 1, where no Slepian-Wolf coding 
is applied, performs the worst. Case 3 outperforms case 2 because the evenly distributed burden in case 3 avoids the possible early drain of energy in case 2 where there is always one fixed node sending more data than the other. However, the performance gap between cases 1, 2, and 3 diminishes as $h$ increases, since as $h$ increases, the difference between $H\left(X_{1}\right), H\left(X_{2}\right), H\left(X_{1} \mid X_{2}\right), H\left(X_{2} \mid X_{1}\right)$ and $H\left(X_{1}, X_{2}\right) / 2$ becomes smaller.

The numerical optimization of $T_{1}, T_{2}, R_{1}$, and $R_{2}$ provides the best performance. However, an iterative numerical optimization in the $\mathbb{R}^{4}$ domain is exceedingly complicated in most WSN applications, where energy is a limited resource. Moreover, when the distribution of the source and/or the profile of the channel are time-varying, periodic updates on $T_{1}, T_{2}, R_{1}$, and $R_{2}$ make iterative numerical optimizations even more costly. The analytical results in (5) allow us to jointly optimize $T_{1}, T_{2}, R_{1}$ and $R_{2}$ at a considerably lower computational cost.

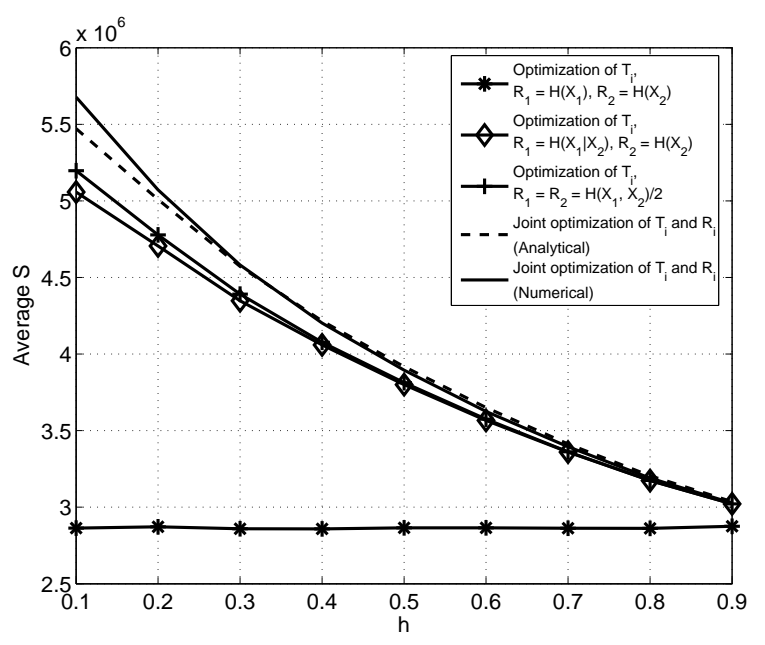

Fig. 3. Comparison of the performance in fusion-centerlimited clusters.

To look at the fusion-center-limited case, we assume that the sensor nodes' residual energy are $E_{1}=E_{2}=100 \mathrm{~J}$, and the fusion center residual energy $E_{0}=0.1 \mathrm{~J}$. Fig. 3 shows the comparison of the performance of the five cases. The analytical results provide a performance that is almost as good as the numerical optimizations, and there is a performance gain over the fixed source coding rate cases (cases 1-3), especially at small $h$ where $X_{1}$ and $X_{2}$ are highly correlated. Our analytical approximations provide a near-optimal performance in a fusion-center-limited cluster.

Comparing Fig. 3 and Fig. 2, the optimization of $R_{1}$ and $R_{2}$ in in clusters with no fusion center energy constraint is more important than in fusion-center-limited clusters. As shown in Fig. 2, in clusters with no fusion center energy constraint, the analytical optimization provides a gain of as much as $101 \%$ on the gathered data over fixed-rate Slepian-Wolf coding cases (cases 2 and 3); while in fusion-center-limited clusters, as shown in Fig. 3 the most gain that the analytical optimization can achieve is merely $8 \%$. This is because, in clusters with no fusion center energy constraint, the total available transmission time duration is determined by the sensor nodes, and an inefficient Slepian-Wolf coding scheme would accelerate the drain of energy at the sensor nodes and thereby reduce the total available transmission time duration. However, in fusion-center-limited clusters, the total available transmission time duration is mainly determined by the fusion center energy storage, and it is not affected by the adopted Slepian-Wolf coding scheme as much as in clusters with no fusion center energy constraint. Thus, when the sensor nodes' energy is limited while the fusion center energy storage is abundant, an optimized distributed source coding scheme becomes crucial.

\section{CONCLUSIONS}

In this paper, we provide analytical results to maximize the lifetime of a 2-source Slepian-Wolf coding cluster with energy constraints and Slepian-Wolf coding constraints. The optimization parameters are source coding rates and transmit durations of both source nodes. The optimal transmit powers of source nodes are also found. For the case where there is no active energy constraint at the fusion center, we have found an exact optimal solution. For the case where there is an energy constraint at the fusion center, near-optimal solutions are obtained. The optimization in the case where there is no energy constraint at the fusion center results in a very significant gain in lifetime.

\section{REFERENCES}

[1] A. Majumdar, K. Ramachandran, and I. Kozintsev, "Distributed Coding for Wireless Audio Sensors," Proceedings of the IEEE Workshop on Applications of Signal Processing to Audio and Acoustics, Oct. 2003.

[2] J. Chou, D. Petrovic, and K. Ramachandran, "A Distributed and Adaptive Signal Processing Approach to Reducing Energy Consumption in Sensor Networks," Proceedings of the IEEE Twenty-Second International Annual Joint Conference (INFOCOM), 2003.

[3] F. Oldewurtel, M. Foks, and P. Mahonen, "On a Practical Distributed Source Coding Scheme for Wireless Sensor Networks," Proceedings of the IEEE Vehicular Technology Conference (VTC), May 2008.

[4] Stephen P. Boyd and Lieven Vandenberghe, Convex Optimization, Cambridge University Press, Cambridge, UK, first edition, 2004.

[5] R. Cristescu, B. Beferull-Lozano, and M. Vetterli, "Networked Slepian-Wolf : Theory, Algorithms, and Scaling Laws," IEEE Trans. on Information Theory, vol. 51, no. 12, pp. 4057-4073, Dec. 2005.

[6] Thomas M. Cover and Joy A. Thomas, Elements of Information Theory, Wiley-Interscience, NJ, second edition, 2006. 